\title{
Estimating Network Offered Load for Optical Burst Switching Networks
}

\author{
Przemyslaw Lenkiewicz ${ }^{2}$, Marek Hajduczenia ${ }^{1,3}$ \\ Mário M. Freire ${ }^{2}$, Henrique J.A. da Silva ${ }^{3}$, and Paulo P. Monteiro ${ }^{1,4}$ \\ ${ }^{1}$ Siemens S. A., Research and Development Department, \\ Rua Irmãos Siemens, $n^{\circ}$ 1, 2720-093 Amadora, Portugal \\ ${ }^{2}$ Department of Informatics, University of Beira Interior, \\ Rua Marquês d'Avila e Bolama, 6201-001 Covilhã, Portugal \\ ${ }^{3}$ Faculdade de Ciências e Tecnologia, \\ Universidade de Coimbra - Pólo II, 3030-290 Coimbra, Portugal \\ ${ }^{4}$ Instituto de Telecomunicações - Pólo de Aveiro, \\ Universidade de Aveiro, 3810-193 Aveiro, Portugal \\ przemek.lenkiewicz@gmail.com, \\ marek.hajduczenia@siemens.com, mario@di.ubi.pt, \\ hjas@ci.uc.pt, paulo.monteiro@siemens.com
}

\begin{abstract}
The Optical Burst Switching (OBS) technology itself is still in the early stage of development and various studies are often performed independently, resulting in difficult comparison between individual data sets (including such controversial studies as burst/packet loss evaluation). To facilitate the future research and evaluation of OBS networks, in this paper we examine relations between standard network parameters and the resulting network offered load, creating a common ground for comparing various simulation results. Means of estimating the resulting network offered load based on parameters describing the network topology and type of traffic are developed and examined for various simulation scenarios (topologies, loads, etc.). It is argued that, given the target offered load value for a given topology, it is always possible to estimate the required idle time which had to be applied in the network nodes, in order to keep the offered network load at the pre-defined level.
\end{abstract}

Keywords: Optical Burst Switching Networks, Network Simulation, Network Offered Load, Network Load Estimation.

\section{Introduction}

The growing interest on OBS networks and the increasing number of available simulation results, conducted using typically custom developed applications, result in a complete chaos when attempting to compare simulation results produced by independent researchers. The situation is further deteriorated by the lack of any common measures for even such basic values as effective and offered network loads, which are required for proper understanding of the operating conditions imposed on the network structure. Furthermore, many simulation results are produced disregarding the establishing of network operating conditions, thereby leading to irreproducible results, which can hardly be compared with any other research in this field. It is hereby 
proposed to adhere to a very simple definition of offered network load, which is one of the prime measures of the OBS network operating conditions and, apart from network topology, link capacity, link length, etc., constitutes an important parameter when comparing various simulation results.

The remainder of this paper is organized as follows. Section 2 includes information about OBS networks and the research conditions. Section 0 presents a description of the simulation results, along with their analysis. Conclusions are presented in section 4, and are followed by literature references in Section 6.

\section{Research Motivation and Methodology}

\subsection{Offered Load Versus Effective Load}

The capacity of any data network can be defined as the amount of traffic that can be transferred through it in a unit of time. For example, assuming that a given network structure has four bi-directional links, each with a bandwidth of $1 \mathrm{Gbit} / \mathrm{s}$, we might easily establish that the capacity of the network in question is $8 \mathrm{Gbit} / \mathrm{s}$. On the other hand, the network traffic load is the amount of traffic that the users generate and try to transfer through the network, producing a certain amount of data transmission events, which require allocation of resources. The network offered load is therefore defined in a straightforward manner as the ratio between the total network traffic load and the network capacity, as indicated by equation (1). It should be noted that, according to this definition, the offered network load can be greater than 1, since users might generate more traffic than the maximum that the network structure can relay. Therefore, such a measure describes very well the network condition, since under light and moderate load conditions (where all or almost all generated traffic can be delivered without imposing packet loss) it will be characterised by a value smaller than 1 , while network overflow (when users attempt to transmit more data than the given network structure can accept within a given time unit) results in an offered load value greater than 1. The network effective load is defined as the ratio between the carried traffic and the network capacity, as expressed by equation (2).

$$
\begin{aligned}
& L_{\text {offered }}=\frac{\sum_{i=1}^{n} L_{i}}{\sum_{j=1}^{m} C_{j}} \\
& L_{\text {effective }}=\frac{\sum_{i=1}^{n} M_{i}}{\sum_{j=1}^{m} C_{j}}
\end{aligned}
$$

In these equations:

- $\quad L_{\text {offered }}$ is the network offered load;

- $\quad L_{\text {effective }}$ is the network effective load;

- $\quad L_{i}$ is the amount of traffic generated by a single user $i$ (out of $n$ ) in a unit of time; 
- $\quad C j$ is the capacity of a single link $j$ (out of $m$ ) in the network (here, for simplicity, we assume that all links have the same capacity);

- $\quad M i$ is the amount of traffic carried over a given link $i$;

- $\quad n$ is the number of active users in the network (producing traffic);

- $\quad m$ is the number of links in the network.

Taking into consideration the previously examined network example with $8 \mathrm{Gbit} / \mathrm{s}$ of raw capacity, and assuming that active users generate $7 \mathrm{Gbit} / \mathrm{s}$, the offered load of the network can be readily estimated as 0.875 . It is therefore clearly visible that, contrary to the network effective load, offered load can significantly exceed 1, leading to congestion and packet loss.

\subsection{Reconfigurable OBS Simulator}

All the following simulation results were obtained using a custom-built, object oriented, event driven simulator of a generic OBS network, with in-built reconfiguration capabilities (based on text configuration files). In this simulator, all the physical components of the OBS network are represented as objects, and the events related to all network elements are processed by simulating the behaviour of said objects. By using a topology description file and standard input characteristics of traffic generation, we were therefore able to observe closely the network operation for a pre-defined period of time. In this way, we could obtain reliable statistics without building an actual OBS network test-bed. All the required system parameters can be set up either through a direct call to proper set-up procedures or through the aforementioned configuration files, including: the network topology, its parameters (user, node, and link properties), and the characteristics / shape / nature of the traffic produced by users. The network description is kept in a topology definition file. The results produced by the OBS simulator have been validated by comparison with those previously reported by other researchers, e.g. [1] . Additionally, prior to the development of this simulator, a number of tests were designed to check the behaviour of the software, once completed. These tests included simulation of various network topologies under varying load conditions, unbalanced load conditions, varying number of users, and irregular topologies. All the tests performed were completed successfully, thereby proving that the software was designed and developed correctly, reproducing all OBS network specific characteristics.

\section{Research}

\subsection{Input Parameters}

Selection of the OBS architectures to be used in the simulations was mainly driven by the requirement of testing various topologies, featuring different number of nodes, links, and throughput. Specifically, the following architectures were included in the evaluations of the proposed model:

- $\quad$ D2T: ring network with 16 nodes (Fig. 1.a);

D3T $(1,15,3)$ and D3T(1,15,5): chordal rings with chord lengths of 3 and 5 (Fig. 1.b and c); 


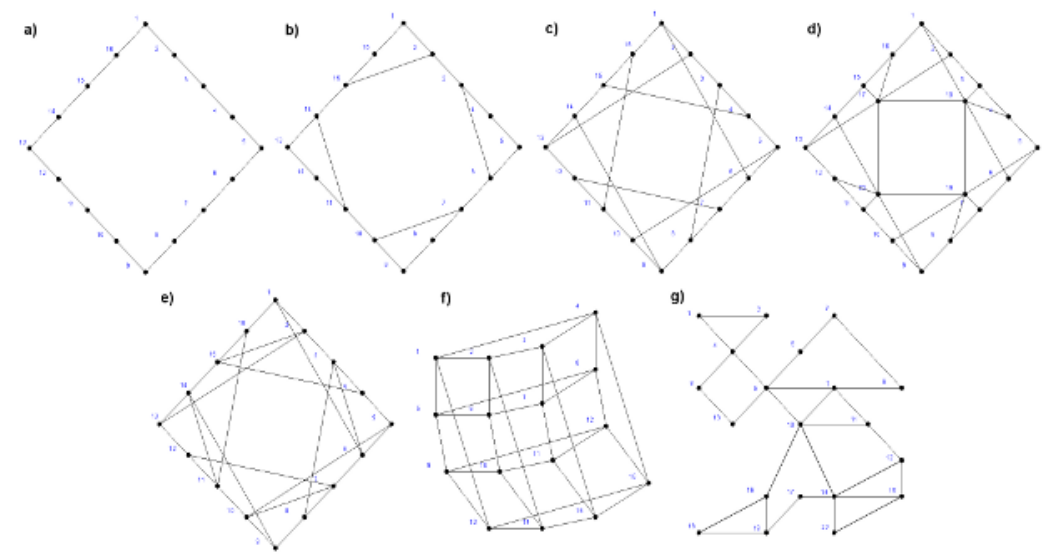

Fig. 1. Network topologies included in the research

- $\quad$ D3T $(1,15,5)$ : chordal ring with 4 additional core nodes (Fig. 1.d);

- $\quad$ D4T $(1,15,3,5)$ : chordal ring with 2 chords (lengths of 3 and 5) (Fig. 1.e);

- MeshTorus 16: mesh-torus network with 16 nodes (Fig. 1.f);

- MeshTorus 25: mesh-torus network with 25 nodes;

- Improvisation: randomly placed 20 nodes with 29 links (Fig. 1.g);

- GEANT: representation of GEANT network (http://www.geant.net);

- Very Simple: ring network with 4 nodes.

To simplify the examination of the resulting data sets, all links were assumed to have the same propagation delay and the same bandwidth. The Dijkstra [2,3] algorithm was used for control packet routing within the OBS network structure. The input traffic fed into the OBS network structure was produced using the standard Poisson random number distribution, and it is typically described using two parameters, namely the average burst length and the average node idle time. The former parameter describes the length of the burst expressed in a common reference time unit (milliseconds in this case, which are converted into picoseconds, selected as a common time reference base for the whole simulation), while the latter one expresses the length of the idle cycle for the given node between generations of two bursts (again expressed in milliseconds, converted into picoseconds for system compatibility). In terms of actual network operation, the aforementioned parameters express the traffic creation intensity. The former one describes the amount of time it takes for a given burst to be transferred from source to destination, while the latter one describes the amount of time it takes for a node to collect sufficient number of packets to meet its burst assembly conditions (depending on the employed burst aggregation scenario).

Each complete simulation scenario included 50 consecutive simulations using the same set of input network parameters. Once completed, the network parameters were altered accordingly, and the simulation cycle was repeated. The average burst size was varied between 150 and 10 milliseconds, with size values decreasing by $20 \%$ of their previous values in each simulation step. Similarly, the average node idle time was varied time between 20 and 1 millisecond, also decreasing by $20 \%$ in each simulation step. In total, 13 simulation steps were performed, producing network offered 
loads ranging from 0.02 to 46 , thus reflecting all possible operating conditions for the OBS network (ultra light, light, moderate, heavy load, and network overload). Further increase/decrease in the network load would not contribute to more detailed analysis of the examined problem, and thus was avoided.

\subsection{Estimation of Basic Approximation Curves}

The estimated network offered load values obtained varied between 0.02 and 46, which represent two extreme network load conditions, when the OBS structure is very lightly loaded (only $2 \%$ of resources used) or flooded with data (46 times the nominal raw network capacity), as shown in Fig 2 (a) and (b). These figures depict the relation between the input simulation parameters, namely average idle time and average burst size, and the resulting offered load. It was observed that it is possible to express the network offered load, with reasonable accuracy, in terms of a power function of the average node idle time and in terms of a linear function of the average burst size. Since these dependencies constitute the grounds for the optimization study presented further on, it was decided to use those simple functions.

Following the aforementioned assumption about mixed power and linear function approximations of the network offered load, let us assume that the network offered load for a particular topology is described by equation (3), which depends only on the average node idle time (6). In order to provide the targeted relation between the network offered load and the pair average node idle time / average burst size (6), a more generic approximation (4) must be produced. Since (3) depends only on the average node idle time, the approximation coefficients $p_{1}$ and $p_{2}$ must be expressed in terms of average burst size by equations (5), where $g$ and $h$ are some generic functions, undefined at this point. In order to fully examine the relations between the network offered load and the pair average burst size / node idle time, several OBS network topologies were examined, with Fig. 2 depicting the obtained offered network load surfaces for a ring network with 16 nodes (Fig. 1.d) and a mesh-torus network with 16 nodes (Fig. 1.f).

$$
\begin{gathered}
L_{\text {offered }}=p_{1} \cdot \hat{T}_{\text {node }}^{p_{2}} \\
L_{\text {offered }}=f\left(\hat{T}_{\text {burst }}, \hat{T}_{\text {node }}\right) \\
p_{1}=g\left(\hat{T}_{\text {burst }}\right), p_{2}=h\left(\hat{T}_{\text {burst }}\right) \\
\hat{T}_{\text {burst }}, \hat{T}_{\text {node }}
\end{gathered}
$$

Each selected network topology was further examined by performing a power function approximation of the measured network offered load values, following equation (3), thereby producing approximation curves similar to those presented in Fig. 3 (only two examples are depicted due to space limitation). Next, the data series were subject to power function regression, producing values of the targeted approximation coefficients $p_{1}$ and $p_{2}$, which were later on collected for each particular topology and depicted against average burst time, as shown in Fig. 4. Fig. 4.(a) presents the relation between the network topology, average burst size, 

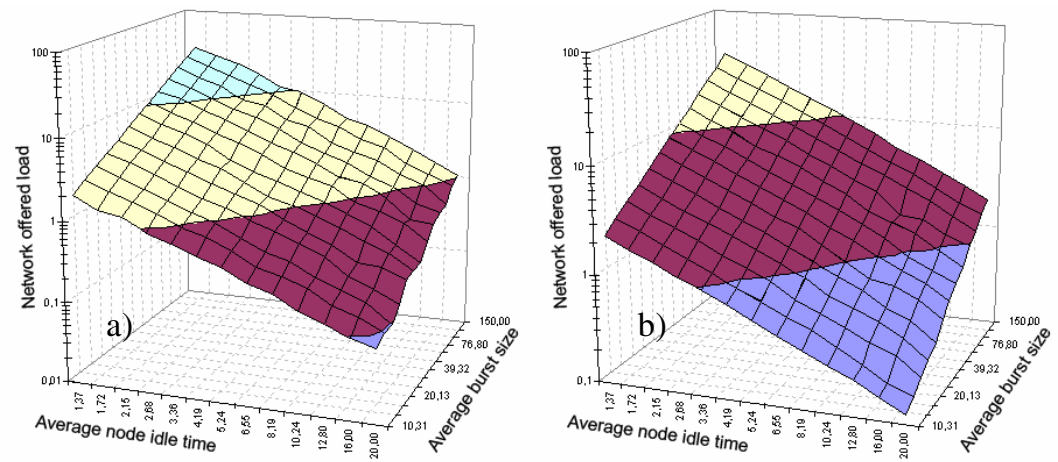

Fig. 2. Relation between network offered load and input traffic parameters in log scale for: (a) a ring network with 16 nodes (Fig. 1.d) and (b) a mesh-torus network with 16 nodes (Fig. 1.f)
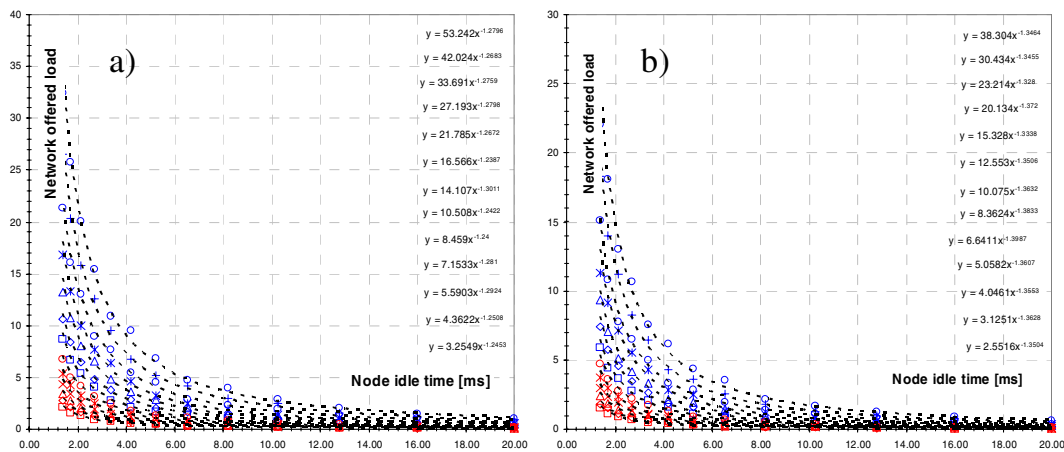

Fig. 3. Network offered load approximation against average node idle time (power function regression (3)) for: (a) a ring network with 16 nodes (Fig. 1.d) and (b) a mesh-torus network with 16 nodes (Fig. 1.f)

and approximation coefficient $p_{1}$ in equation (3), while Fig. 4.b presents the corresponding relation though this time for the coefficient $p_{2}$ in equation (3). It is visible that the coefficient $p_{1}$ exhibits a power function character when plotted against average burst size, while the coefficient $p_{2}$ has a quasi linear (constant) value with tiny fluctuations around the average value.

Next, when examining the relations in Fig. 4, it is immediately visible that the approximation coefficients $\mathrm{p} 1$ and $\mathrm{p} 2$ exhibit significant correlation with the network topology, which was expected, when comparing the examples of the network offered load surfaces for two different topologies, depicted in Fig. 2. Since the main goal of this paper is to produce a topology-independent, generic formula describing the offered network load as a function of the average node idle time and burst size (both expressed in milliseconds), it is necessary to establish a relation between each obtained topology-dependent curve and a particular examined OBS network topology. 
First, the resulting relations (depicted in Fig. 4) had to be described as a function of the average burst length, by using a power function regression in the case of the p1 coefficient (7) and a strictly linear function regression in the case of the p2 coefficient (8). Then, the obtained regression coefficients had to be described as a function of the examined OBS network topology. Since there is no observable relation between the regression coefficients and any straightforward network parameters (number of nodes, edge nodes, links etc), two new network measures had to be devised and examined, namely:

- node-link density, which is hereby defined as the total number of nodes in the given topology divided by the number of links interconnecting the said nodes - equation (9), where $N_{\text {node }}$ and $N_{\text {link }}$ are numbers of nodes and links, respectively;

- network diversity, which is hereby defined as the difference between the total number of links in the network and the network diameter - equation (10), where $D$ is the network diameter;
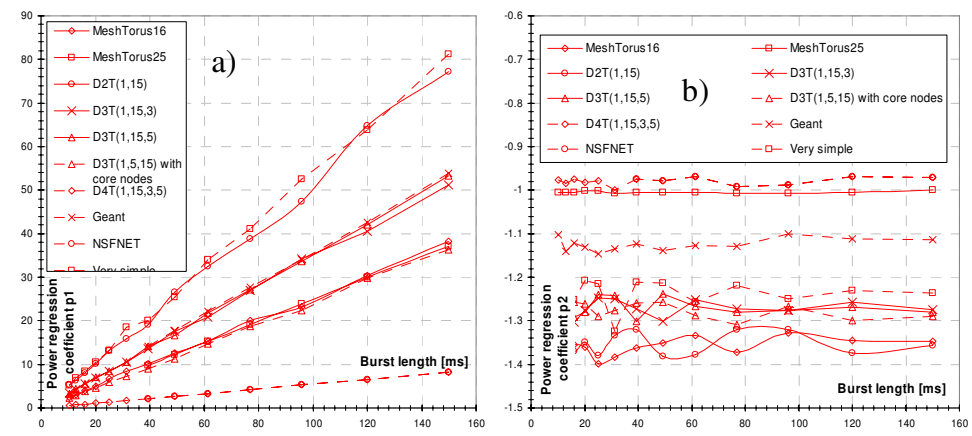

Fig. 4. Established relations between: (a) power approximation coefficient $p_{1}$ and (b) power approximation coefficient $p_{2}$ as a function of average burst length, for examined topologies

$$
\begin{gathered}
p_{1}=p_{1}^{\prime} \cdot\left(\hat{T}_{\text {burst }}\right)^{p_{1}^{\prime \prime}} \\
p_{2}=p_{2}^{\prime} \cdot\left(\hat{T}_{\text {burst }}\right)+p_{2}^{\prime \prime} \\
\rho_{\text {node/link }}=\frac{N_{\text {node }}}{N_{\text {link }}} \\
\vartheta=N_{\text {link }}-D \\
p_{1}^{\prime}, p_{1}^{\prime \prime} \\
p_{2}^{\prime}, p_{2}^{\prime \prime}
\end{gathered}
$$



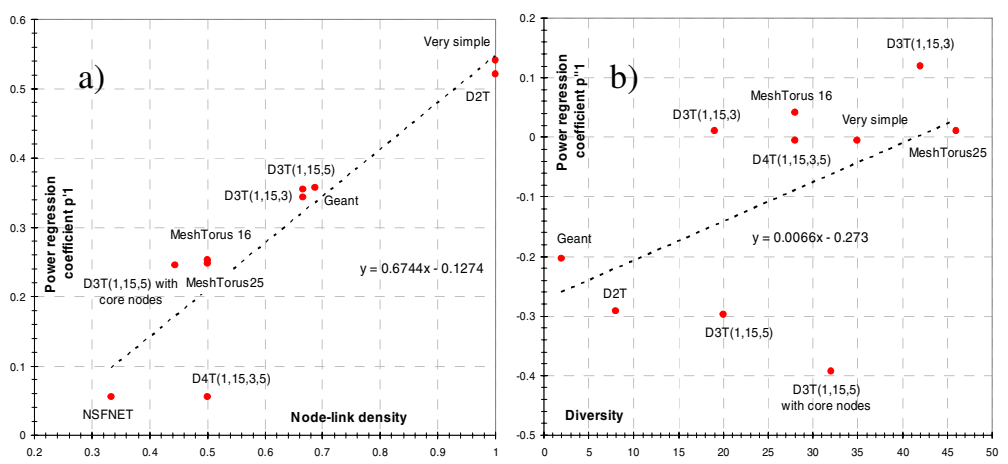

Fig. 5. Power function regression coefficients (11) (Fig. 4.a) and their relation with (a) nodelink density (9), for the first regression coefficient, and (b) network diversity (10), for the second regression coefficient
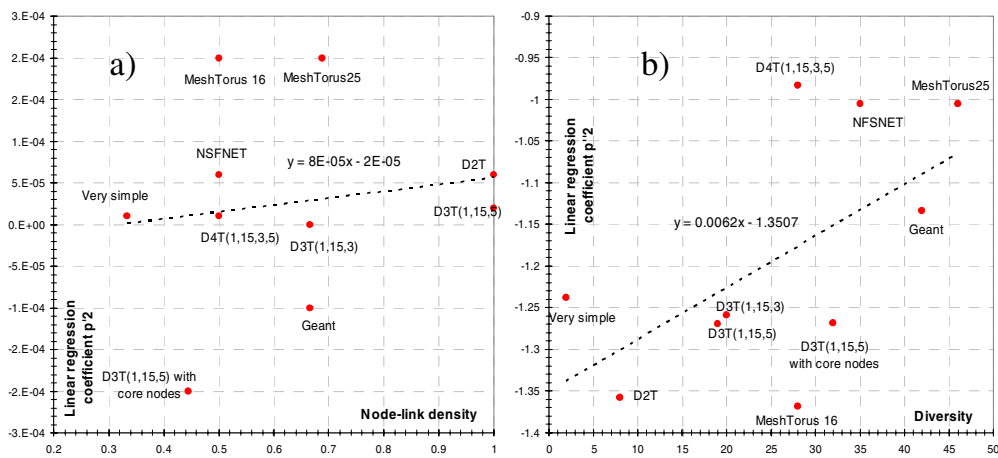

Fig. 6. Linear function regression coefficients (12) (Fig. 4.b), and their relation with (a) nodelink density (9), for the first regression coefficient, and (b) network diversity (10), for the second regression coefficient. The first coefficient was disregarded $\left(<10^{-4}\right)$.

It was observed that the first of the regression coefficients (11) follows the abovedefined node-link density coefficient (9) in a strictly linear manner, showing virtually no discrepancy for the examined topologies (see Fig. 5.(a)). The second of the regression coefficients (11) proved to be virtually topology independent and maintain a quasi-constant value as a function of the network diversity parameter (10) (see Fig. 5.(b)). In the case of the $p_{2}$ coefficient, the linear regression equation (8) proved to be virtually independent of the average burst size (see Fig. 4.(b)), and thus the first of the linear regression coefficients (12) is expected to have a value close to 0 , which is further confirmed by Fig. 6.(a), where the values of this regression coefficient have a magnitude smaller than $10^{-4}$. Further considerations will therefore omit this parameter due to its marginal value. The second of the regression coefficients (12) was plotted as a function of the network diversity parameter (10) (Fig. 6.b), and exhibits a linear dependence, though this time with significant discrepancy from the approximated value. 
The final equations that model the relation between the network topology description and the initial power law regression coefficients for network offered load are (17) and (18), while (13) to (16) are partial equations obtained from the respective regressions. It is worth noting that only $p_{1}$ depends on the average burst size, while $p_{2}$ seems to depend only on the network diversity parameter.

$$
\begin{gathered}
p_{1}^{\prime}=0.6744 \cdot \rho_{\text {node } / \text { link }}-0.1274 \\
p_{1}^{\prime \prime}=0.066 \cdot \vartheta-0.273 \\
p_{2}^{\prime}=0 \\
p_{2}^{\prime \prime}=0.0062 \cdot \vartheta-1.3507 \\
L_{\text {offered }}=\left\{\begin{array}{c}
\left.0.6744 \cdot \rho_{\text {node /link }}-0.1274\right) \cdot\left(\hat{T}_{\text {burst }}\right)^{0.066 \cdot \vartheta-0.273} \\
p_{2}=0.0062 \cdot \vartheta-1.3507 \\
\left(0.6744 \cdot \rho_{\text {node /link }}-0.1274\right) \cdot\left(\hat{T}_{\text {burst }}\right)^{0.066 \cdot \vartheta-0.273} . \\
\left(\hat{T}_{\text {node }}\right)^{0.0062 \cdot \vartheta-1.3507}
\end{array}\right\}
\end{gathered}
$$

The final expression linking the offered network load, average burst time, and average node idle time, is therefore (19), where the node link density and network diversity parameters describe each particular OBS network topology in a unique manner.

\subsection{Result Validation / Precision}

Once the final equation (19) linking the offered network load, average burst time, and average node idle time was established, its approximation accuracy required evaluation. The validation process consisted of calculating the network offered load surfaces (similar to those depicted in Fig. 2), comparing them with the simulation results, and presenting the resulting discrepancy (if any) between the calculated and simulated network offered load for all types of examined OBS topologies. The simulation process comprised 100 cycles per each data point (a pair of average burst size and node idle times), resulting in approximately 17,000 simulations per single examined topology, thereby providing a sufficient sample size to calculate and examine confidence intervals.

First, the D3T $(1,15,5)$ network depicted in Fig. 1.d was examined, by producing the average network offered load surface depicted in Fig. 7.a. Fig. 7.b presents the size of the $95 \%$ confidence interval, proving that the obtained simulation results were consistent and repetitive. Fig. 8.a depicts the calculated network offered load surface, based on (19), with the discrepancy between the calculated and measured network offered load shown in Fig. 8.b. 

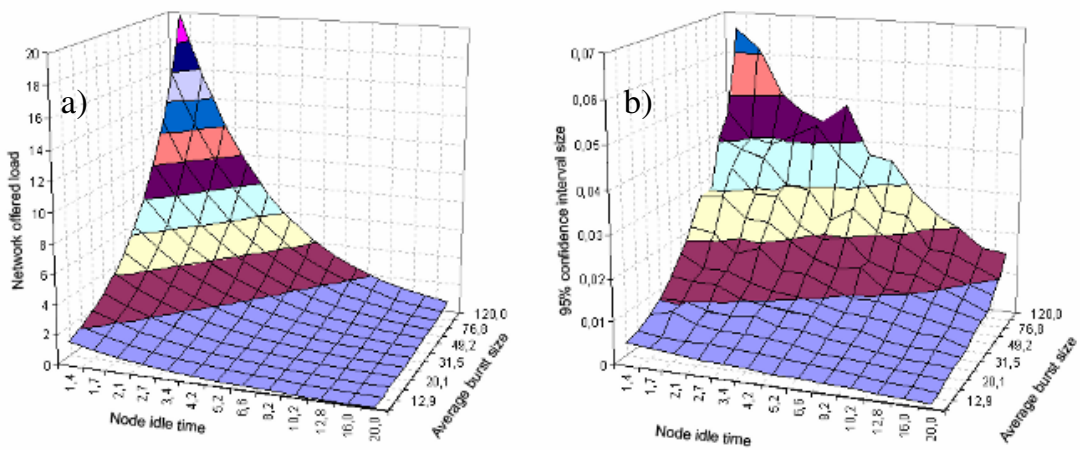

Fig. 7. (a) Simulated average network offered load for D3T $(1,15,5)$ network (averaged over 100 simulations per data point) and (b) Size of the $95 \%$ confidence interval
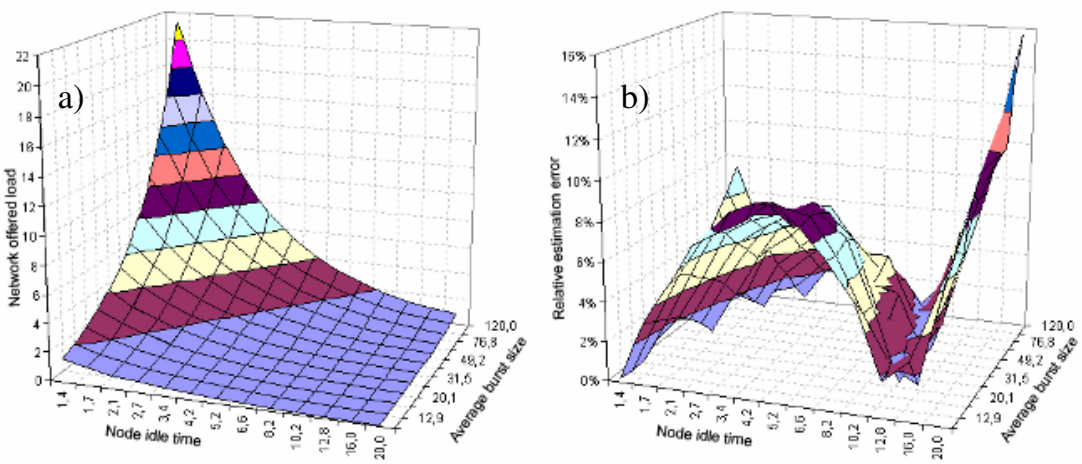

Fig. 8. (a) Calculated network offered load for D3T(1,15,5) network and (b) Relative difference between the simulated and calculated network offered load

It is a straightforward observation that the calculated network offered load surface is consistent with the simulated one, apart from the high overload area, where the estimation error is significant and exceeds $10 \%$. However, it must be noted that a standard network is typically never subject to such high loads, exceeding the raw nominal capacity more than 20 times. The area of interest (offered network load ranging from 0 to 5) is approximated with very good quality, exhibiting estimation errors below $8 \%$. Additionally, it must be noted that the observed estimation errors for very low offered loads (below 0.02 for very long average burst length, above $100 \mathrm{~ms}$ ) results from finite simulation length. It was additionally observed that the said error diminishes as the simulation cycle length is increased, though that resulted in excessive simulation process time (in excess of 5 days for single average node idle time) and therefore was not further explored.

Next, a different network type was examined, namely a Mesh-Torus network with 16 nodes, as depicted in Fig. 1.f. The same simulation process conditions were applied also in this case, producing a complete set of characteristics. The average 

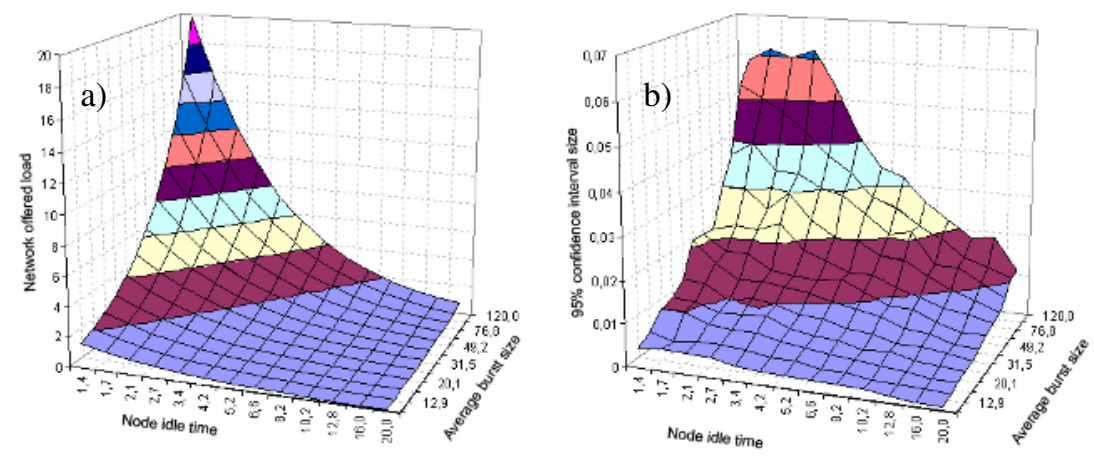

Fig. 9. (a) Simulated average network offered load for a Mesh-Torus network with 16 nodes (averaged over 100 simulations per data point) and (b) Size of the $95 \%$ confidence interval
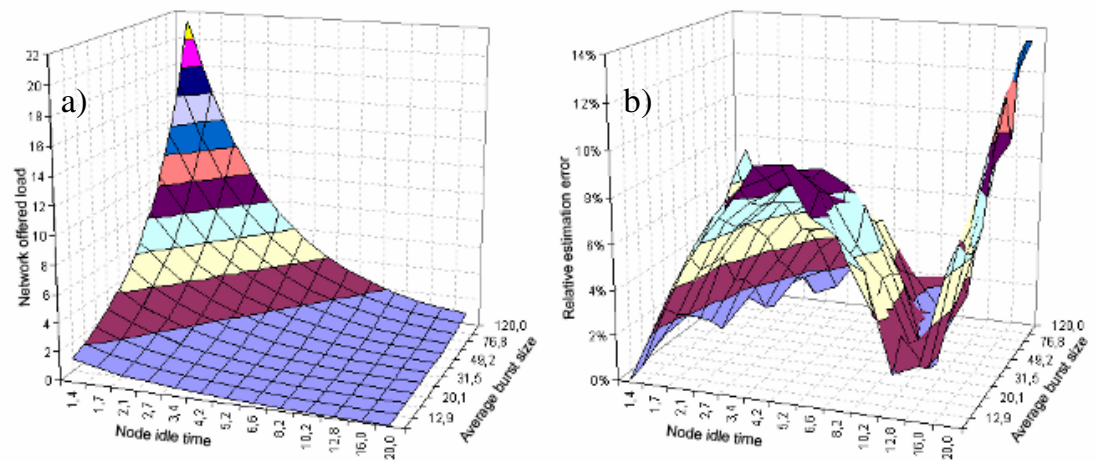

Fig. 10. (a) Calculated network offered load for a Mesh-Torus network with 16 nodes and (b) Relative difference between the simulated and calculated network offered load

network offered load surface is depicted in Fig. 9.(a), while Fig. 9.(b) shows the size of the $95 \%$ confidence interval, proving that the obtained simulation results were consistent and repetitive.

Fig. 10.(a) depicts the calculated network offered load surface, based on (19), with the discrepancy between the calculated and simulated network offered loads shown in Fig. 10.(b). Again, it is possible to observe that the calculated network offered load surface is consistent with the simulation results, with the mean estimation error below $8 \%$. Slightly higher discrepancies are observed for very low network offered load with large average burst size (above $100 \mathrm{~ms}$ ), the reasons for which were explained before. It is therefore concluded that the derived generic network offered load estimation equation (19), depending only on the generalized network topology description and input parameters such as average burst size and average node idle time, is consistent with the simulation results and can be used successfully to estimate the input values for said parameters, prior to performing any simulations. 


\section{Conclusions and Future Work}

In this paper, we present a straightforward method to estimate the resulting network offered load based on such OBS network simulator parameters as network topology type and input traffic parameters (average node idle time and average burst size). The accuracy of this method was validated by comparing measured and calculated network offered load surfaces, producing estimation errors below $8 \%$ for moderate and high load while estimation error in excess of $15 \%$ was noticed only for ultra low network load with very large average burst size (in excess of $100 \mathrm{~ms}$ ), where it is argued that increased simulation time leads to better convergence though extends the simulation process exorbitantly. However, since network offered load generalization is possible, assuming that a simple parameter (namely network topology name and description) is provided, the results of the corresponding simulations using various custom-built OBS simulators can have a common ground for comparison, once the network offered load matches. Additionally, using the approximation model we present in this paper, it is always possible to define the network operation point (average burst size and node idle time), and thus have a priori settings for the input traffic generator.

There is also room for future work, including improving the approximation precision of the general, topology-independent equation, and searching for new ways to describe differences between individual network topologies. Moreover, our study assumed that all links in the network share the same raw bandwidth (data rate and number of channels), which does not necessarily need to hold true in the case of real OBS networks. It is therefore imperative to evaluate the impact of varied link capacity on the network offered load. Other research issues for this topic include also OBS signalling algorithms, varied propagation delays (link lengths) and varied burst/packet loss probabilities.

\section{Acknowledgments}

This work has been financially supported by Fundação para a Ciência e a Tecnologia (FCT), Portugal, through the grant contract SFRH/BDE/15524/2004 and through CONDENSA Project contract POSC/EEA-CPS/60247/2004 and by Siemens S.A. COM RD1 R, Portugal.

\section{References}

1. J. J. C. P. Rodrigues, M. Freire, and P. Lorenz: Performance Assessment of Signaling Protocols with One-Way Reservation Schemes for Optical Burst Switching Networks. High Speed Networks and Multimedia Communications (2004)

2. T. H. Cormen, C. E. Leiserson, R. L. Rivest, and C. Stein: Introduction to Algorithms, Section 24.3: Dijkstra's algorithm. pp.595-601, 2nd ed: MIT Press and McGraw-Hill, (2001)

3. E. W. Dijkstra: A note on two problems in connection with graphs, Numerische Mathematik. (1959) vol. 1, pp. 269 - 271 